\title{
A Self-biased 3D Tunable Helical Antenna in Ferrite LTCC Substrate
}

\author{
Farhan Abdul Ghaffar \\ Electrical Engineering Program \\ King Abdullah University of Science and Technology (KAUST) \\ Thuwal, Saudi Arabia \\ farhan.ghaffar@kaust.edu.sa
}

\author{
Atif Shamim \\ Electrical Engineering Program \\ King Abdullah University of Science and Technology (KAUST) \\ Thuwal, Saudi Arabia \\ atif.shamim@kaust.edu.sa
}

\begin{abstract}
A ferrite LTCC based helical antenna which also provides magneto-static bias for its frequency tuning is presented in this work. The 3D helical-cum-bias winding design avoids the use of large external electromagnets which are traditionally used with ferrite based tunable antennas. This reduces the overall size of the design while making it efficient by getting rid of demagnetization effect experienced at the air-to-ferrite interface. RF choke and DC blocking capacitor, required to isolate the RF and DC passing through a single structure, are integrated within the multi-layer Ferrite LTCC substrate. Magnetostatic and microwave simulations have been carried out for the design optimization. The prototype antenna demonstrates a tuning range of $10 \%$ around $13 \mathrm{GHz}$. An optimized design with an air cavity is also presented which reduces the biasing power requirement by $40 \%$.
\end{abstract}

\section{INTRODUCTION}

Modern wireless devices require multiple transceivers and antennas to cater for various applications operating in different frequency bands. A low cost and size efficient solution can be a frequency tunable antenna that can cover these frequency bands. Ferrite is a suitable candidate for tunable antenna implementation. However, present ferrite based antennas are bulky due to the use of large external electromagnets [1]. Moreover these designs are inefficient due to the demagnetization effect experienced at the air-to-ferrite interface [2]. These issues of bulk ferrite substrates can be resolved by using a multilayer ferrite technology such as Low Temperature Co-fired Ceramic (LTCC) [3], where the external magnets can be replaced by embedded bias windings.

A tunable patch antenna in ferrite LTCC technology is reported in [4], where the magnetostatic bias for the antenna substrate is provided by using embedded solenoid windings underneath the ground plane of the antenna. The antenna demonstrates a tuning range of $10 \%$ around its center frequency. Although the reported tunable antenna provides encouraging results it needs a separate bias winding for generation of magnetostatic fields. An interesting alternative can be a helical structure which can acts as an antenna and the bias winding at the same time.

\section{HelicAl ANTENNA/Bias Winding DESIGN}

\section{A. Initial Design}

A helical antenna has a similar structure as a solenoid bias winding. Therefore it can be used as a dual-purpose element i.e. an antenna and a bias source at the same time. To accomplish this it is essential to physically isolate the DC (low) frequency excitation and RF signal. For this purpose a DC block capacitor and RF chokes are required. Instead of using these components externally one can integrate these elements with the antenna in the same substrate. ESL 40012, a multilayer ferrite LTCC tape system, is used for this work. The antenna design concept is shown in Fig. 1. First, the helical antenna is designed for the center frequency of $13 \mathrm{GHz}$ in ten layers of ESL $40012\left(\varepsilon_{\mathrm{r}}=14.6\right)$ using CST Microwave Studio. Through simulations, antenna circumference of $7 \mathrm{~mm}(\mathrm{r}=1.15 \mathrm{~mm})$ and a conductor width of $0.75 \mathrm{~mm}$ is deemed suitable, which provides a broadside radiation pattern, a gain of $2.1 \mathrm{dBi}$ and an impedance bandwidth of $450 \mathrm{MHz}$. The low value of the antenna gain is due to the high dielectric constant of the substrate. After optimized for its RF performance, the helical is simulated in CST EM Studio (magnetostatic simulator) to study its magnetostatic performance. These simulations are important to relate the current excited in the antenna and the magnetization produced in the substrate.

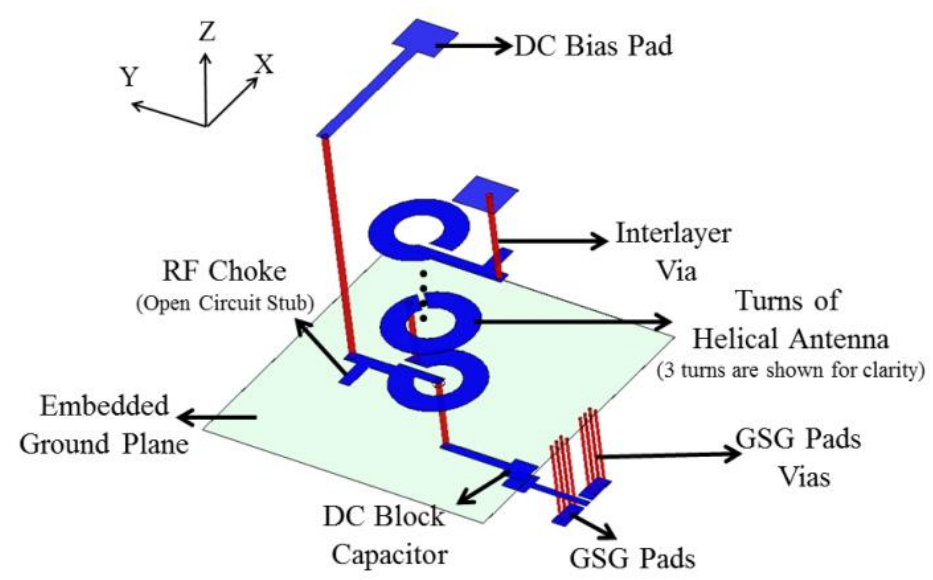

Fig. 1. Helical Antenna Design Concept

The fabricated antenna is shown in Fig. 2 (a). Impedance measurements show that in the unbiased state the antenna operates at $13.1 \mathrm{GHz}$ while for a maximum current of 1100 $\mathrm{mA}$ the center frequency of the antenna decreases to 11.8 $\mathrm{GHz}$, showing a tuning range of $1.3 \mathrm{GHz}$. This correlates well with the simulated tuning range of $1 \mathrm{GHz}$, as shown in Fig. 2(b). Gain and radiation pattern measurements of the antenna 
reveal that the performance is not much affected during the biasing of the substrate.

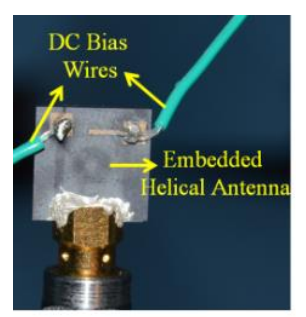

(a)

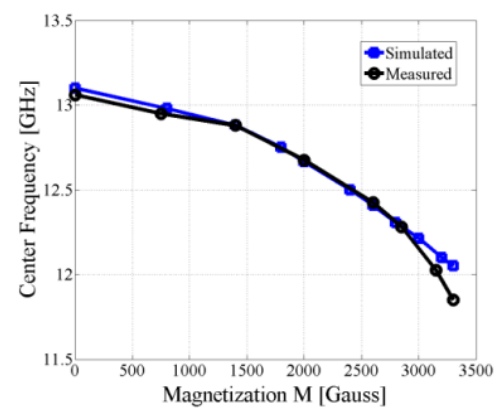

(b)
Fig. 2. (a) Fabricated helical antenna (b) Simulated and measured frequency tuning of helical antenna

\section{B. Optimized Design}

The drawback of this helical-cum bias winding design is that it utilizes $1100 \mathrm{~mA}$ to provide a tuning range of $1.3 \mathrm{GHz}$. This current value results in a large power dissipation of 1.2 $\mathrm{W}$. In the first prototype, the antenna is completely surrounded by the ferrite material. As a result, the magnetostatic fields produced by the windings travel into the substrate away from the antenna and are wasted. If the ferrite material surrounding the helical antenna can be removed, this can help concentrate the magnetostatic fields around the antenna because the reluctance of air is much higher than that of the ferrite substrate. The modified design, based on this concept, is shown in Fig. 3. The air cavities have been created around the antenna to concentrate the magnetostatic fields near the antenna (shown in white color).

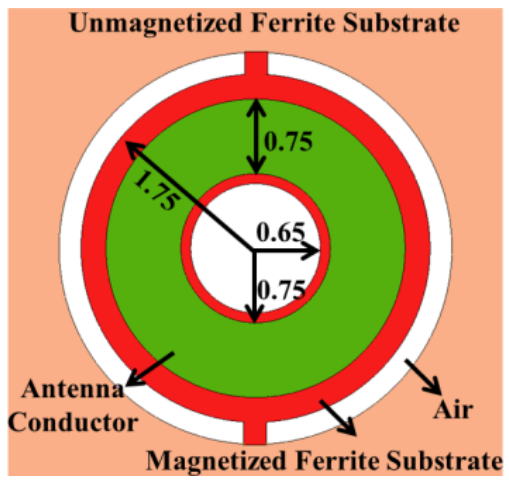

Fig. 3. Optimized helical antenna design (dimensions are in $\mathrm{mm}$ )

From the magneto-static simulations it is observed that a magnetization of $3250 \mathrm{G}$ can be produced from this modified design by using a current of $800 \mathrm{~mA}$. This means that a current reduction of $30 \%$ is achieved as compared to the initial design. This current results in a power consumption of $640 \mathrm{~mW}$, which is $40 \%$ lower as compared to the initial design. After magneto-static simulations, microwave simulations have been performed for the optimized design. The results are shown in Fig. 4 (a). In the unbiased state the antenna operates around $13.5 \mathrm{GHz}$ which tunes down to 12.5 $\mathrm{GHz}$ for a magnetization of $3250 \mathrm{G}(800 \mathrm{~mA})$. So the tuning range of $1 \mathrm{GHz}$ obtained from the initial design is maintained here but with $40 \%$ lower power consumption. The simulated radiation pattern of the optimized antenna is shown in Fig. 4(b). The antenna radiates parallel to its axis (i.e. Z-axis) which shows performance in the axial mode. The radiation performance of the antenna remains constant with and without the magnetostatic bias which is desired from this design.

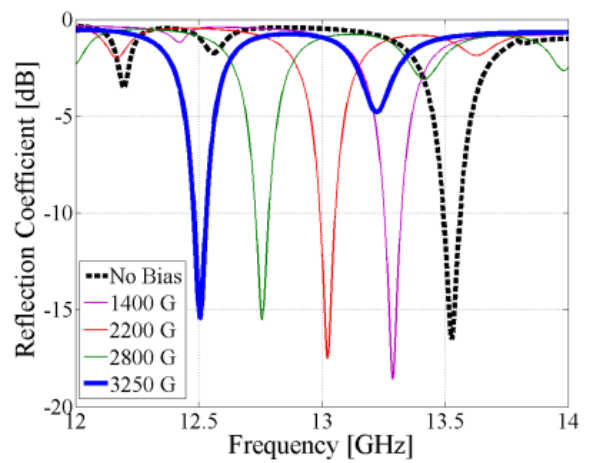

(a)

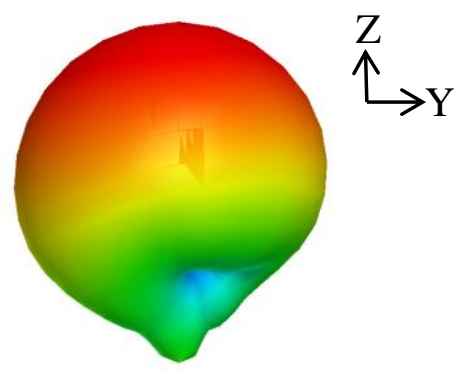

(b)

Fig. 4. Simulated (a) S11 of the optimized antenna (b) 3D radiation pattern

\section{CONCLUSION}

A ferrite LTCC based tunable helical antenna providing bias for the substrate is presented in this work. The design has been optimized using two different simulators and integrated with RF chokes and DC block capacitor in the same package. A tuning range of $10 \%$ with a maximum current of $1100 \mathrm{~mA}$ is obtained from the design. In the end it is shown that an air cavity can be created around the antenna which can reduce the DC power consumption by $40 \%$ as compared to the initial design while maintaining the same tuning performance.

\section{REFERENCES}

[1] L. R. Tan, R. X. Wu, C. Y. Wang, Y. Poo, "Magnetically tunable ferrite loaded SIW antenna," IEEE Antennas and Wireless Propagation Letters, vol. 12, pp. 273-275, 2013.

[2] A. Petosa, E. K. Mongia, M. Cuhaci and J. S. Wight, "Magnetically tunable ferrite resonator antenna", IEEE Electronic Letters, vol. 30, no. 13, pp. 1021-1022, 1994.

[3] M. Franz, K. Makarovic, S. Luftl, A. Maric, I. Atassi, M. Hrovat, N. Blaz, Radosavljevic, G., "Characterization and binder burnout studies of ferrite LTCC tapes," 36th International Spring Seminar on Electronics Technology (ISSE), , pp.17-23, 2013.

[4] F. A. Ghaffar, J. Bray, Atif Shamim, "Theory and design of a tunable antenna on a partially magnetized ferrite LTCC substrate", IEEE Transactions on Antennas and Propagation, Vol. 62, no. 3, pp. 1238-1245, 2014. 